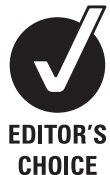

${ }^{1}$ Osteoporosis Screening Unit, Guy's Hospital, London, UK ${ }^{2}$ Department of Chemical Pathology, St Thomas' Hospital, London, UK

\section{Correspondence to} Dr Geeta Hampson, Department of Chemical Pathology, 5th Floor, North Wing, St Thomas Hospital, London SE1 7EH, UK geeta.hampson@kcl.ac.uk

Accepted 29 June 2011 Published Online First 6 September 2011

\title{
The pathogenesis, diagnosis, investigation and management of osteoporosis
}

\author{
Sunita K Sandhu, ${ }^{1}$ Geeta Hampson ${ }^{1,2}$
}

\section{ABSTRACT}

With an increasingly ageing population, osteoporosis and osteoporosis-related fractures is fast becoming an important public health problem placing a considerable economic burden on health service resources. This does not account for the substantial pain, disability and indeed mortality incurred after a fracture, particularly a hip fracture. Osteoporosis is a systemic skeletal disorder which results from an imbalance in bone remodeling. This leads to a reduction in bone strength and increased susceptibility to fracture. It affects up to 1 in 2 women and 1 in 5 men. In the past 2 decades, there have been significant advances in bone biology which have helped in the understanding of the pathogenesis of osteoporosis and have led to improved therapies. In developing strategies for fracture prevention, it is important to identify those individuals with the highest fracture risk who will require pharmacological intervention. Treatment is aimed at fracture prevention and includes modification of general lifestyle factors which have been linked to fractures in epidemiological studies and ensuring optimum calcium and vitamin $D$ intake as adjunct to active anti-fracture therapy. A number of drugs are now approved for the treatment of osteoporosis. This review article will describe the pathogenesis of osteoporosis and focus on the methods currently in use for the identification of patients at high fracture risk and will highlight their usefulness and limitations. The existing anti-fracture pharmacotherapies and those in development will be reviewed. Assessment of their effectiveness including the use of biochemical markers of bone turnover in this clinical context will be reviewed.

\section{INTRODUCTION}

Osteoporosis is a systemic skeletal disorder which results from an imbalance in bone remodelling, leading to a reduction in bone strength, with microarchitectural disruption and skeletal fragility, increasing fracture susceptibility. ${ }^{12}$ Bone strength is a function of both bone density and quality. Bone quality refers to a composite of factors that determine bone resistance to fracture such as its micro-

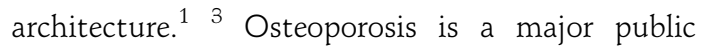
health and financial problem. In the UK, one in two women and one in five men will suffer a fracture after the age of 50, with an annual cost to the health services of around $£ 2$ billion. ${ }^{4-7}$ Hip fracture is the most serious consequence of osteoporosis and, like other osteoporotic fractures, is associated with increased mortality risk and reduced quality of life, with up to $50 \%$ of patients left with a permanent impaired mobility. ${ }^{8-11}$ A recent meta-analysis showed that among older men and women, the rate of death from any cause is increased by a factor of 5 to 8 during the first 3 months after a hip fracture. ${ }^{12}$ Increased mortality risk persisted for 5 to 10 years following all low trauma fractures in a prospective cohort study of men and women aged $>60$ years. ${ }^{13}$ Therefore, prevention of fractures is the primary goal of intervention.

\section{PATHOGENESIS}

Osteoporosis can occur because of (1) failure to achieve peak bone mass and (2) excessive bone resorption and/or decreased bone formation during remodelling. All these processes are likely to contribute, in varying degrees, to osteoporosis.

\section{Achievement of peak bone mass}

Attainment of peak bone mass is of prime importance in preventing osteoporosis and subsequent fractures in adulthood. Hip fracture can be reduced by $30 \%$ with an increase in peak bone mass of $10 \%$. Genetic factors are major determinants of peak bone mass and bone loss, contributing as much as $80 \%$ to peak bone mass variability based upon twin studies. Genome-wide association studies have identified several genetic variants that regulate bone mass, including low-density lipoprotein receptorrelated protein 5 (LRP5), sclerostin (SOST), osteoprotegerin (OPG), oestrogen receptor 1 and receptor activator of NF- $\kappa \beta$ (RANK) pathway genes. ${ }^{14}$ So far, 20 genes have been identified that predispose to osteoporosis, but they each contribute to only a small amount of the genetic variance in susceptibility to the disease. ${ }^{15}$ This could be because genome-wide association studies are geared towards identification of common variants of small effects rather than rare variants of large effects. Further work is in progress to determine rare polymorphisms in other candidate genes which could have larger effects. ${ }^{16}$ However, at present, not enough is known about the genetics of osteoporosis to influence clinical decision making. Bone mass accretion in childhood and early adulthood is also influenced by hormonal status, particularly oestrogen. Environmental factors such as nutrition, exercise and smoking play important roles in achievement of peak bone mass. ${ }^{17} 18$ It is now known that modulation of peak bone mass can occur during intrauterine life and is affected by maternal nutrition, smoking and level of exercise. ${ }^{19}$

\section{Imbalance of bone resorption and bone formation}

In adulthood, the bone remodelling process is pivotal for bone health maintenance as it repairs areas of microdamage. This is a cellular process involving coordinated actions of osteoclasts (bone resorbing cells) and osteoblasts (bone forming cells), which form the bone multicellular unit. The 
OPG/RANK and its ligand (RANKL) are the most important mediators of osteoclast activity, whereas LRP5 is one of the most important mediators of osteoblast activity. ${ }^{20}{ }^{21}$ Other cells contribute to the bone remodelling process and include the terminally differentiated osteoblasts, osteocytes and vascular elements forming the bone remodelling compartment. Bone resorption is stimulated through production of RANKL by cells of the osteoblastic lineage. Binding of RANKL to its cognate receptor RANK results in activation of complex intracellular pathways including NF- $\kappa \beta$, which results in induction of osteoclastogenic genes. OPG acts as a decoy receptor, preventing RANKL binding to RANK. Proteins called Wnt's activate the LRP5 pathway, which stimulates bone formation and inhibits bone resorption. A variety of inhibitors of LRP5 signalling have been identified including SOST, which is produced by osteocytes and likely acts as a mediator of the effects of mechanical loading on the skeleton. ${ }^{22}$

The OPG/RANKL ratio is a key factor in maintenance of normal bone turnover and bone mass/strength. Numerous hormones, growth factors (TGF- $\beta$, IGF-1, BMP2), cytokines (IL-1, IL-6, TNF- $\alpha$, prostaglandins E2) and drugs influence the expression of OPG/RANKL and therefore bone turnover ${ }^{23-26}$ (table 1). It is well established that imbalance in bone remodelling at the menopause is due to oestrogen deficiency. ${ }^{27}$ In men, although there is a slower reduction in serum testosterone with advancing age, bioavailability diminishes progressively, particularly after age 80 , resulting in bone loss, though much of the effect of testosterone is mediated by aromatisation to oestradiol. ${ }^{28}$

Although it seems likely that increased bone resorption has the greatest impact on bone loss and fracture risk, ${ }^{29}{ }^{30}$ impaired bone formation in response to increased bone resorption rate is an important component of the pathogenesis of osteoporosis. This is thought to be due to a reduction in the number of osteoprogenitor/pre-osteoblastic cells and/or an age-related defect in their proliferative and differentiation abilities. With increasing age, bone formation falls more than bone resorption, presumably due to a preferential differentiation of bone marrow stromal cells into adipocytes rather than osteoblasts.

Age and menopause-related bone losses are clearly important factors for osteoporosis, with genetic factors likely explaining the wide variations in skeletal integrity in older persons of the same age. ${ }^{31}$ In addition, age-related factors such as visual acuity, muscle strength, poor balance and drugs that affect balance will interact with bone density in an important way to determine fracture risk.

\section{DIAGNOSIS AND INVESTIGATION}

A major problem of osteoporosis management is that majority of those at high fracture risk are not diagnosed or treated, despite availability of safe and effective diagnostic tools and therapies. ${ }^{32}{ }^{33}$ This paucity of care applies to both women and men, although more evident in men.

Table 1 Effect of several hormones on OPG/RANKL ratio

\begin{tabular}{llll}
\hline Hormones & OPG & RANKL & OPG/RANKL \\
\hline Oestrogen & $\uparrow$ & $\downarrow$ & $\uparrow$ \\
Glucocorticoid & $\downarrow$ & $\uparrow$ & $\downarrow$ \\
Parathyroid hormone* & $\downarrow$ & $\uparrow$ & $\downarrow$ \\
1,25(OH) $)_{2}$ vitamin D & $\uparrow$ & $\downarrow$ & $\uparrow$
\end{tabular}

$\downarrow$, Decrease; $\uparrow$, Increase.

*Continuous PTH exposure results in decrease OPG/RANKL ratio; however, intermittent exposure does not lead to significant alteration in OPG/RANKL ratio, while stimulating markers of bone formation.

$\mathrm{OPG}$, osteoprotegerin; RANKL, receptor activator of NF- $\kappa \beta$ ligand.

\section{Measurement of BMD}

Diagnosis of osteoporosis is based on measurement of bone mineral density (BMD), using dual X-ray absorptiometry (DXA), which is considered the gold standard. However, a personal history of fragility fracture is consistent with a clinical diagnosis of osteoporosis, regardless of $\mathrm{BMD}$, in the absence of other causes of skeletal fragility. BMD results are reported as a comparison to a sex-matched young healthy adult (T-score) or a sex-matched and age-matched healthy population (Z-score) and is expressed in standard deviations. The WHO has defined osteoporosis as a T-score of less or equal to -2.5 and osteopenia as a T-score between -1.0 and $-2.5 .^{34}$ The T-score criteria are used for postmenopausal women and men aged 50 years or older. Outside this demographic group, the T-score is less accurate as a predictive tool for fracture risk. The International Society for Clinical Densitometry recommend using the Z-score instead of $\mathrm{BMD}$ to be measured in premenopausal women and men younger than 50 years of age, with a score less than or equal to -2.0 indicative of a bone density that is below the expected range for age. ${ }^{35} \mathrm{BMD}$ is measured at both the spine and hip. The forearm ( $33 \%$ radius) is recommended if the hip and/or spine cannot be measured or interpreted, in hyperparathyroidism and in obese patients who are over the weight limit for DXA table. $\mathrm{BMD}$ is a specific predictor of fracture risk as demonstrated in several studies in men and women. Individuals with T-scores below -2.5 have progressively higher fracture risk. However, patients with $\mathrm{T}$-scores above -2.5 may still be at high risk of fracture. It has been estimated that over $50 \%$ of patients with hip fractures and $35 \%$ with vertebral fractures have a T-score above $-2.5^{36-38}$ Hence, a combination of $\mathrm{BMD}$ and clinical risk factors for fracture predicts fracture risk better than $\mathrm{BMD}$ alone.

\section{Assessment of clinical risk factors and fracture risk}

Fracture is the outcome of multiple risk factors, and this multiplicity should be taken into account in assessment of fracture risk for an individual. Several important clinical risk factors have been identified through epidemiological studies, including age, falls and history of fragility fractures (box 1). ${ }^{39-41}$ In addition, several disorders and drugs can lead to increased bone loss and are important secondary causes of osteoporosis. Differential diagnosis includes a thorough medical history, physical examination and a range of investigative tests which may be case-dependent (box 2).

In order to assist clinicians in their clinical management process, the WHO task force has developed and introduced a country-specific Fracture Risk Assessment Tool (FRAX), based upon data collected from large international cohorts in which clinical risk factors, $\mathrm{BMD}$ and fractures were evaluated. The tool combines $\mathrm{BMD}$ measurement and clinical risk factors to derive a 10-year probability of hip fracture or major osteoporotic fracture (combined hip, spine, humerus or wrist), thus allowing identification of individuals at high fracture risk. ${ }^{42}$ Those with probabilities above the intervention threshold will require pharmacological intervention (National Osteoporosis Guideline Group). Absolute risk assessment is now recognised as a preferred approach to guide treatment decision as opposed to $\mathrm{RR}$, which is a comparison between different risk levels and not a reflection of the individual's actual risk. The FRAX tool is particularly useful in identification of patients with osteopenia who are at higher risk of fracture. Though the FRAX algorithm is useful in assessing an individual's absolute fracture risk and hence individualising treatment, it has some limitations which prevent it from being the gold standard. First, it has been designed for postmenopausal women and men older than 


\section{Box 1 Clinical risk factors for osteoporosis and fractures}

$\mathrm{Age}^{*}$

- Sex*

- Ethnicity

- Femoral neck bone mineral density*

- Body weight*

- History of prior fractures*

- Height*

- Parental history of hip fracture*

- Current smoking*

- Glucocorticoid therapy* $\dagger$

- Alcohol consumption (3 or more units per day)*

- Rheumatoid arthritis*

- History of falls

- Secondary osteoporosis (eg, hyperthyroidism, hypogonadism or premature menopause, malabsorption, chronic liver disease, inflammatory bowel disease) ${ }^{*}$

- Medications (eg, immunosuppressants, antiseizure medications, heparin, chemotherapy)

*Clinical risk factors included in the FRAX algorithm

†Past or present exposure to prednisone equivalent dose of $5 \mathrm{mg}$ or more for more than 3 months.

40 years of age who have not previously been on bone protective treatment. Second, it does not take into account dose-responses for several risk factors including glucocorticoid use. Third, falls or increased propensity to falls is not included in the algorithm. Fourth, femoral neck BMD only is used to compute fracture risk; therefore, absolute fracture risk may not be accurate in those

Box 2 Laboratory evaluation for secondary causes of osteoporosis

Initial laboratory tests

- Renal profile (Urea, Creatinine)

- Calcium, phosphorus, magnesium

- Liver function tests

- Full blood count

- 25 Hydroxyvitamin D

- Thyroid stimulating hormone

- Parathyroid hormone

Additional laboratory tests if indicated

- Sex hormones (Testosterone, Oestradiol, LH, FSH)

- Coeliac serology

- Serum/Urine protein electrophoresis

- Erythrocyte sedimentation rate

- 24-h Urine calcium/Creatinine

- Bone turnover markers

- 24-h Urine free cortisol

- Prolactin

- Iron studies

- Serum tryptase and histamine levels

- Homocysteine

- Rheumatoid factor

- Skin biopsy for connective tissue disorders

$\mathrm{LH}$, luteinising hormone; $\mathrm{FSH}$, follicular stimulating hormone. patients who have lowest $\mathrm{BMD}$ at the spine. In addition, it is country-specific and has to be used cautiously in countries where there is no representation.

\section{CLINICAL MANAGEMENT/THERAPEUTIC INTERVENTIONS}

Treatment is aimed at reducing future fracture risk and should be tailored to the individual patient. The emphasis should be on early recognition and effective management of patients at high fracture risk.

\section{Non-pharmacological intervention}

This includes modification of general lifestyle factors, such as a balanced diet containing calcium and vitamin $\mathrm{D}$, smoking cessation and avoidance of heavy alcohol use. A regular exercise routine should be encouraged, including weight-bearing and muscle-strengthening exercises. ${ }^{43}{ }^{44}$ In a Cochrane analysis of 18 randomised-controlled trials examining the effectiveness of exercise therapy in preventing bone loss in postmenopausal women, aerobics, weight bearing and resistance exercises were all effective on the BMD of the spine. ${ }^{45}$ Walking was effective on both $\mathrm{BMD}$ of the spine and the hip and should be recommended as it is the easiest and simplest program to implement. Longterm studies to determine fracture data are required. Falls prevention is integral in fracture prevention. Interventions suitable for falls reduction has been summarised in a Cochrane review and include an exercise program incorporating balance, gait and strength training. ${ }^{46}$ There is also evidence to support correcting of vision impairment as part of a multifactorial approach. ${ }^{47}$

The role of calcium and vitamin D supplementation in reducing falls and fractures is not clear. A reduction in either calcium intake or absorption and/or vitamin $\mathrm{D}$ deficiency/insufficiency leads to secondary hyperparathyroidism, which contributes to accelerated bone loss in the elderly. ${ }^{48} \mathrm{~A}$ meta-analysis concluded supplementation for people aged 50 years or more conferred a reduced risk of any fracture of $12 \%(\mathrm{RR}=0.88,95 \% \mathrm{CI}$ 0.83 to 0.95 ), the greatest effect being with a daily dose of $1200 \mathrm{mg}$ calcium and $800 \mathrm{IU}$ vitamin D. ${ }^{49}$ The antifracture effect of vitamin $\mathrm{D}$ is more pronounced in the vitamin-D-deficient institutionalised elderly and involves, in part, its effect on muscle strength and therefore in falls prevention. ${ }^{50} 51$ Vitamin D deficiency impairs neuromuscular function, increasing falls risk (figure 1). As calcium was given along with vitamin $\mathrm{D}$ in many of the positive trials, it has become routine clinical practice to replete both calcium and vitamin D. Indeed, all trials of antifracture therapies have been carried out in subjects given both calcium and vitamin $\mathrm{D}$ as adjunct. It can be concluded therefore that the antifracture abilities of these drugs apply to calcium-replete and vitamin-D-replete individuals. ${ }^{52}$

What constitutes optimum vitamin $\mathrm{D}$ status is still a subject of debate and is defined in some studies as a serum 25-hydroxyvitamin D (25OHD) concentration of $>50$ or $75 \mathrm{nmol} / 1^{53} 54$ Several criteria have been used to define 'sufficient' $250 \mathrm{HD}$ level, including the level associated with optimal suppression of parathyroid hormone (PTH), greatest calcium absorption, highest $\mathrm{BMD}$, lowest rates of bone loss, lowest rates of falling and lowest fracture rates. Defining optimal $25 \mathrm{OHD}$ concentration is further complicated by differences in assay methodologies and standardisation which needs addressing to assist design of population supplementation strategies. Based on available evidence, it has been suggested that although estimates of serum $25 \mathrm{OHD}$ level needed for optimal suppression of PTH varied widely, optimal vitamin D status clusters in the 75 to $80 \mathrm{nmol} / \mathrm{l}$ range 


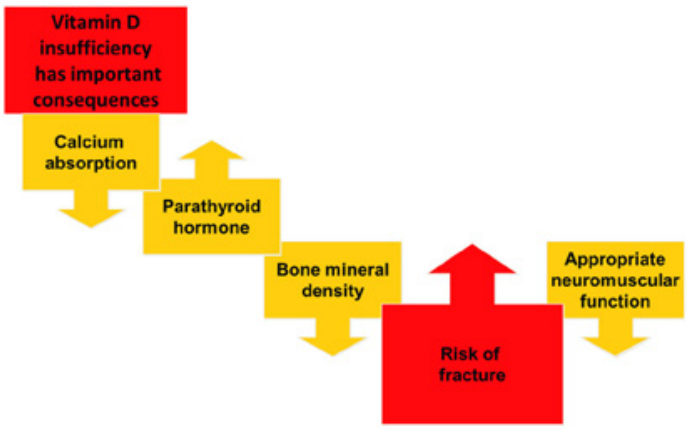

Figure 1 Consequences of vitamin $D$ deficiency/insufficiency. The reduction in intestinal calcium absorption associated with vitamin $D$ inadequacy triggers the release of parathyroid hormone, which mediates the mobilisation of calcium from bone, resulting in a reduction in bone mineral density. This homoeostatic response to vitamin $D$ inadequacy may increase the risk of fractures. Another important consequence of vitamin $D$ inadequacy is a decrease in neuromuscular function.

with a lower risk of fracture at serum levels $>75 \mathrm{nmol} / \mathrm{l}^{55} 56$ Calcium absorption is considered to be impaired under conditions of vitamin $\mathrm{D}$ deficiency, though it is uncertain at what vitamin $\mathrm{D}$ status, calcium absorption is fully normalised. In an analysis of two randomised cross-over studies, mean calcium absorption was $65 \%$ higher at serum $250 \mathrm{HD}$ levels averaging $86.5 \mathrm{nmol} / \mathrm{l}$ than at levels averaging $50 \mathrm{nmol} / 1 .{ }^{57}$ A recent histomorphometry study of 675 German adults showed a surprisingly high incidence of osteomalacia but no evidence of osteomalacia with $250 \mathrm{HD}$ concentrations $>75 \mathrm{nmol} / \mathrm{l}$, suggesting this may be an appropriate cut-off for optimal bone health. ${ }^{58}$ Serum $250 H D$ below these concentrations are highly prevalent in the UK, particularly in the elderly where a significant proportion have serum $250 \mathrm{HD}$ concentrations $<25 \mathrm{nmol} / 1$ which constitutes vitamin $\mathrm{D}$ deficiency. Though there is a consensus to maintain $25 \mathrm{OHD}$ levels $>25 \mathrm{nmol} / \mathrm{l}$ in the UK, there is uncertainty regarding the strength of evidence for aiming for substantially higher $250 \mathrm{HD}$ concentrations. ${ }^{59}$
Supplementation with calcium in osteoporosis prevention is currently in question following a recent meta-analysis which showed calcium supplements to be associated with about a $30 \%$ increased risk of myocardial infarction. ${ }^{60}$ In contrast, there appears to be an association between a low 25OHD status and increased CVD risk. ${ }^{61}$ A meta-analysis showed decreased total mortality rates with vitamin $\mathrm{D}$ supplementation, with no change in RR with the addition of calcium supplements. ${ }^{62}$ The main concern with excessive vitamin $\mathrm{D}$ is hypercalciuria and hypercalcaemia. However, there is a relatively large therapeutic window for vitamin $\mathrm{D}$, with hypercalcemia not reported with blood $250 \mathrm{HD}$ levels $<220 \mathrm{nmol} / 1$ and generally not reported until blood levels reach $500 \mathrm{nmol} / \mathrm{l}^{63}$

We would recommend aiming for a serum 250HD of $75 \mathrm{nmol} / 1$ in high-risk population, such as the housebound and institutionalised elderly; however, this may be difficult to achieve with the current recommended vitamin $\mathrm{D}$ intake of 800-1000 IU, which is more a maintenance dose. On average, $25 \mathrm{OHD}$ rises by approximately $0.7 \mathrm{nmol} / 1$ for each microgram (40 IU)/day of supplemental vitamin D3 to reach a plateau at about 8 weeks. ${ }^{64}$ With regard to calcium, we would recommend a daily intake of 1000-1200 mg daily, with supplements suggested only if dietary intake is inadequate. Compliance is an issue; hence, side effects of supplements need to be taken into account. With calcium supplements, side effects such as constipation may reduce overall compliance with all medications and hence limit treatment effectiveness.

\section{Pharmacological intervention}

Significant advances in bone biology in the past two decades have helped in understanding the pathogenesis of osteoporosis, leading to better therapies. A number of drugs are now approved for treatment of osteoporosis (table 2), but there is a lack of high-quality head-to-head drug comparison trials to determine the relative efficacy of individual drugs. A systematic review of 76 randomised trials and 24 meta-analyses confirmed efficacy of multiple agents compared with placebo in fracture prevention. ${ }^{65}$ The bisphosphonates (alendronate, risedronate, zoledronate) and oestrogen reduced risk of vertebral, non-vertebral and hip

Table 2 Therapeutic agents available for osteoporosis, mode of action and fracture efficacy

\begin{tabular}{|c|c|c|}
\hline & Mechanism of action & Fracture efficacy \\
\hline $\begin{array}{l}\text { Bisphosphonate (etidronate, } \\
\text { ibandronate, alendronate, } \\
\text { risedronate, zoledronate) }\end{array}$ & $\begin{array}{l}\text { Antiresorptive: Reduce rate of bone turn-over and } \\
\text { maintain or improve skeletal architecture. } \\
\text { Inhibit farnesyl pyrophosphate synthase, resulting } \\
\text { in reduced resorptive activity of osteoclasts and } \\
\text { accelerated apoptosis. Rank order of potency is } \\
\text { zoledronate }>\text { risedronate }>\text { ibandronate }>\text { alendronate. }\end{array}$ & $\begin{array}{l}\text { Reduce vertebral fractures by } 50-70 \% \text {, non-vertebral fractures by } 30 \% \\
\text { and hip fractures by } 40 \%{ }^{*} \\
\text { Based on bone turnover markers, maximum effect in } 3 \text { to } 6 \text { months. } \\
\text { Effect on clinical vertebral fractures and non-vertebral fractures at } \\
6-36 \text { months depending on agent used ( } 6 \text { months for risedronate, } \\
12-24 \text { months for alendronate and } 36 \text { months for zoledronate based } \\
\text { on pooled, post hoc analyses. }\end{array}$ \\
\hline
\end{tabular}

Selective Estrogen Receptor Modulator (SERM): for example Raloxifene

Recombinant Human Parathyroid Hormone (PTH)

Strontium Ranelate

Inhibit bone resorption.

Anabolic: stimulates bone formation by reducing osteoblastic apoptosis and increasing osteoblast numbers and differentiation.

Inhibit bone resorption and increase bone formation

Tibolone

Testosterone

Denosumab
Synthetic steroid with oestrogenic, androgenic, and progestagenic properties.

Increase bone formation.

Blocking antibody to Receptor activator of $\mathrm{NF}-\kappa \beta$ Ligand (RANKL) which inhibits osteoclast formation and bone resorption.
Reduce vertebral fracture by $35 \%$ with no decrease in non-vertebral and hip fracture in a meta-analysis.

Reduces vertebral and non-vertebral fractures. Decrease vertebral fractures by $65 \%$ and non-vertebral by $54 \%$.

Reduce vertebral fractures by $40 \%$, and to a lesser extent non-vertebral fractures by $16 \%$ in meta-analysis. Decreased hip fracture by $36 \%$ in post hoc subgroup analysis in women aged 74 years or older.

Reduce vertebral fractures by $45 \%$ and non-vertebral by $26 \%$ in postmenopausal women with improvements in BMD.

Demonstrated to improve BMD in men with hypogonadism. Effects on fracture reduction unclear.

Reduce vertebral fractures by $68 \%$, non-vertebral by $20 \%$ and hip fractures by $40 \%$ in postmenopausal women, associated with increases in BMD and reciprocal decreases in markers of bone resorption 
fractures. However, the Women's Health Initiative, concluded the benefits of hormone replacement therapy (HRT) such as fracture prevention and colon cancer, were outweighed by the risks of breast cancer, coronary heart disease, stroke and thromboembolism, ${ }^{66}$ thus greatly diminishing use of oestrogen in prevention and treatment of osteoporosis. There is, however, a more favourable risk/benefit ratio of HRT in postmenopausal women $<60$ years of age and in women with early menopause. In this group, HRT is recommended by the National Osteoporosis Society (UK) particularly if menopausal symptoms are present, provided there are no risk factors for breast cancer, stroke, heart disease or venous thromboembolism.

Bisphosphonates are synthetic analogues of pyrophosphate which have a high affinity for bone mineral and reduce osteoclastic activity. All bisphosphonates are approved for treatment in postmenopausal osteoporosis with alendronate, risedronate and zoledronate approved for men and glucocorticoid-induced osteoporosis. In the absence of randomised trial data that ibandronate reduces hip fracture risk, alendronate or risedronate are the initial choices for oral bisphosphonates, ${ }^{67} 68$ with alendronate recommended as first choice based on the National Institute of Clinical Excellence (NICE) guidelines, as there are generic versions available making it cost effective. Intravenous zoledronate is used for individuals with gastrointestinal intolerance to or those who have failed treatment with oral bisphosphonates as is ibandronate. There has been a decrease in mortality by about $28 \%$ demonstrated with zoledronate following hip fractures. ${ }^{69} 70$

The association between bisphosphonate use and the rare complication of osteonecrosis of the jaw has been described recently, mainly in patients receiving high-dose bisphosphonates for metastatic cancer and undergoing invasive dental procedures. ${ }^{71} 72$ The risk is low in patients treated for osteoporosis, in the range of 1 in 10000 to 10000 patient years. Nevertheless, patients receiving oral bisphosphonates should have good oral hygiene and see their dentists regularly. In addition, there have been case reports of association of bisphosphonate use with atypical femoral fractures. ${ }^{73}$ In some of these cases, bone biopsies were done and showed severely suppressed bone turnover and delayed healing. ${ }^{74}$ However, there is no definitive evidence with recent reports suggesting no relationship between bisphosphonate and fractures, warranting ongoing investigation. ${ }^{75} 76$ More recent studies have shown a possible association between oral bisphosphonates and increased risk of oesophageal cancer, mainly in patients who have been on bisphosphonates for more than 5 years, though this remains unproven. 7778

Optimal duration of bisphosphonate therapy remains uncertain. There is evidence of ongoing fracture risk reduction if treatment is continued at least for 10 years in patients on alendronate ${ }^{6779}$ and 7 years in patients on risedronate, ${ }^{68}$ with no new safety concerns. However, it is becoming routine clinical practice to recommend a 'drug holiday' (time off bisphosphonate) in patients on long-term bisphosphonate due to potential concerns that long-term suppression of bone turnover may eventually lead to adverse events. The duration of therapy and length of holiday should be individualised, taking into account fracture risk and binding affinity for the bisphosphonate used, with lower affinity agents having a shorter residence time in bone if treatment is stopped. Rank order for binding affinity is zoledronate $>$ alendronate $>$ ibandronate $>$ risedronate. ${ }^{80}$ Based on available evidence, discontinuing therapy after 5 years appears not to be harmful. ${ }^{79} 81$ Because of the long skeletal retention time of bisphosphonates, antifracture benefits still persist despite discontinuation for 12-18 months. However, in patients with a high risk of fracture, it is recommended that a non-bisphosphonate treatment be commenced, such as raloxifene or teriparatide during the holiday from the bisphosphonate. $^{82}$

Other available antiresorptives include the selective oestrogen receptor modulators such as raloxifene, which is somewhat less effective than bisphosphonates, though direct comparison trials are lacking. ${ }^{83}$ Raloxifene is associated with an increased risk of venous thromboembolic events, particularly during the first 4 months of treatment, and it can cause or exacerbate vasomotor symptoms associated with menopause. While there are data to suggest raloxifene may be effective in men, it is not currently recommended for use in men. ${ }^{84}$ In the UK, raloxifene is not recommended for primary prevention of postmenopausal osteoporosis but can be used in secondary prevention as alternative treatment to patients intolerant to or who have contraindications to bisphosphonate.

Strontium ranelate has weak effects on bone resorption and bone formation. However, its mechanism of action is not fully understood, and it is likely that its antifracture efficacy is mediated by its effects on bone material properties rather than its rather weak effect on skeletal remodelling. ${ }^{85}$ A meta-analysis of four trials concluded that there is reasonable evidence strontium ranelate is effective for reducing the risk of vertebral fractures and, to a lesser extent, non-vertebral fractures in women. ${ }^{86}$ A small increase in the risk of venous thromboembolism was seen in clinical trials. Strontium has not been studied in men. In the UK, strontium is recommended as an alternative to bisphosphonates in the prevention/treatment of osteoporosis if patients are unable to comply with administration of are intolerant or have a contraindication to the bisphosphonates.

Intermittent administration of the bone anabolic agent, recombinant human parathyroid hormone (PTH: full length 1-84 or fragment 1-34) stimulates bone formation, which results in improvement in trabecular numbers and connectivity and increased cortical thickness and bone strength. ${ }^{87}$ Larger increases in bone mass, particularly at the lumbar spine, are seen with intermittent PTH than with antiresorptives. ${ }^{88} 89$ Duration of treatment is limited to 24 months. Candidates for treatment are those with very low $\mathrm{BMD}$, at very high risk of fracture and who have failed previous therapy with antiresorptives. Recombinant PTH is also approved for men and postmenopausal women receiving glucocorticoids who are at increased risk of fracture. Sequential therapy with bisphosphonate is required following cessation of PTH.

For women, treatment agents include tibolone, a synthetic steroid used for osteoporosis management in postmenopausal women in some countries. ${ }^{90} 91$ For men with osteoporosis and hypogonadism who do not have any contraindications to testosterone therapy, testosterone replacement therapy is beneficial. $^{92-94}$

Newer therapies available include the humanised monoclonal antibody, denosumab. This binds RANKL, thus reducing osteoclastogenesis. Data from the multicenter FREEDOM trial show that treatment with denosumab in 60- to 90-year-old women over 3 years led to statistically significant increases in $\mathrm{BMD}$ and reductions in bone resorption. ${ }^{95}$ It has been shown to reduce the incidence of new vertebral, hip and non-vertebral fractures in postmenopausal women. In the UK, it is approved for both primary and secondary prevention of postmenopausal osteoporosis. $^{96}$

Emerging therapies under investigation for the treatment of osteoporosis include: oral calcium sensing receptor antagonists which leads to a transient rise in endogenous PTH; SOST 


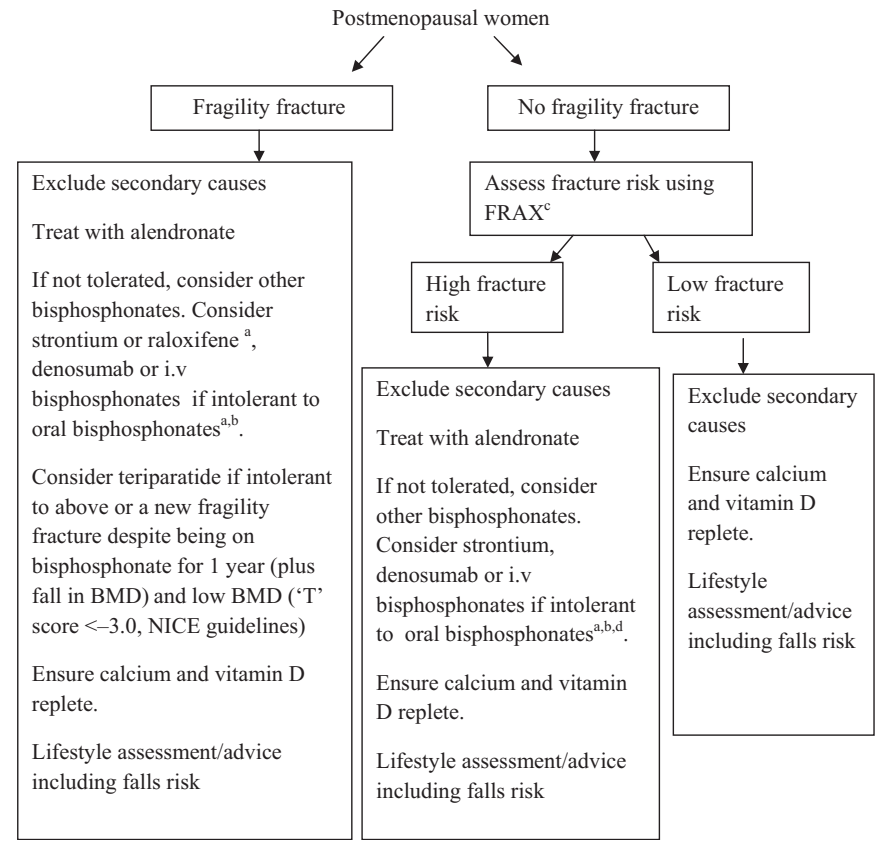

Figure 2 Proposed algorithm for management of osteoporosis in postmenopausal women. (A) Owing to lack of evidence of efficacy in non-vertebral fractures, including hip fractures, we would recommend avoiding ibandronate or raloxifene in patients with non-vertebral fractures or low BMD in non-vertebral sites (not in the NICE guidelines). (B) Consider intravenous bisphosphonates if intolerant to oral bisphosphonates or if treatment failure (not in the NICE guidelines). (C) FRAX not included in the NICE guidelines. (D) Raloxifene is not recommended as a treatment option for primary prevention of osteoporotic fragility fractures in postmenopausal women according the NICE guidelines.

inhibitors (SOST is produced by osteocytes and inhibits bone formation), which increases bone mass in animal studies ${ }^{97}$; integrin antagonists (integrins mediate the adhesion of osteoclasts to the bone surface, an important initial step for bone resorption) ${ }^{98}$; cathepsin- $\mathrm{K}$ inhibitors (cathepsin $\mathrm{K}$ is a protease that may play a role in osteoclast-mediated bone resorption). ${ }^{99}$

Our recommendation for a management approach when treating osteoporosis in postmenopausal women is shown in figure 2. This takes into account the NICE guidelines.

\section{TREATMENT FOLLOW-UP AND MONITORING}

Monitoring response to therapy is important for identifying patients who may require a change in therapy; however, there is no consensus on the optimal strategy for this.

\section{Assessment of changes in BMD}

Some studies suggest that BMD increase during therapy correlate with reduction in fracture risk. ${ }^{100-102}$ A meta-analysis of 12 clinical trials concluded that improvement in spine $\mathrm{BMD}$ with therapy accounts for a predictable but relatively small part of reduction in fracture risk, explaining only $16 \%$ of fracture risk reduction, suggesting contribution of other non-BMD effects of therapy. ${ }^{103}$ In order for a change in $\mathrm{BMD}$ to be considered significant, it should be greater than the 'least significant change' (LSC) for the densitometer in question. Precision error of the DXA scan should be determined and the LSC calculated so as to determine whether an apparent change in $\mathrm{BMD}$ represents a likely biological change or is simply within the range of measurement error. The short-term precision error expressed as coefficient of variation (CV) ranges from $1.9 \%$ to $2.5 \%$ in elderly women at the lumbar spine and hip, respectively. Follow-up $\mathrm{BMD}$ measurements play a role in clinical management. BMD that is stable or improving is evidence for treatment response. Finding of a clinically significant decrease in BMD in a treated patient should trigger additional evaluation for contributing factors, which may include poor adherence to therapy. The International Society for Clinical Densitometry recommends follow-up BMD testing (DXA spine and hip) when the expected change in BMD is likely to equal or exceed the LSC, typically 1-2 years after initiation or change of therapy, with longer intervals once therapeutic effect is established. In conditions associated with rapid bone loss, such as glucocorticoid therapy, testing more frequently is appropriate. ${ }^{104}$

\section{Use of BTMs}

Use of biochemical bone turnover markers (BTMs) in clinical trials has been helpful in understanding mechanism of action of therapeutic agents. A list of commonly used BTMs is shown in table 3 . Their routine use, however, remains a challenge on account of their wide biological and analytical variability, which may be as much as a 7.3-fold difference. ${ }^{105}$ Discordant results are sometimes obtained because of differences in assay techniques, inappropriate collection and timing of sample. The analytical variability can be reduced with automation and improved standardisation between assays and standardisation of sample collection. ${ }^{106}{ }^{107}$ In general, intraindividual variability is lower for serum or plasma than urine markers. Circulating C-terminal cross-linked telopeptide (CTX) can be measured in either plasma or serum and is dependent on the assay used. It is preferable to use plasma when the Roche automated ( $\beta$-crosslaps) assay is used. The CV for plasma CTX and N-terminal cross-linked telopeptide (NTX) is reportedly half that for urine CTX or NTX with CV of $10 \%$ for plasma markers and between $15 \%$ and $25 \%$ for urine markers. The use of well-defined reference ranges can minimise the variability associated with several uncontrollable factors-for example, age, gender, pregnancy, lactation, fracture status and other diseases. Recommendations for establishing reference ranges should be defined and standardised with emphasis on sample size and age range of the population. ${ }^{108}$

The best established clinical use for BTMs is in monitoring treatment efficacy and compliance. Antiresorptive agents such as bisphosphonates, oestrogens, selective oestrogen receptor modulators and denusomab rapidly decrease BTMs. ${ }^{70}{ }^{109-113}$ Significant reductions in bone resorption markers, particularly

Table 3 List of biochemical markers of bone turnover commonly used in clinical practice

\begin{tabular}{llll}
\hline Bone formation markers & Analytical sample & Bone resorption markers & Analytical sample \\
\hline Procollagen type 1 C-terminal propeptide (P1CP) & Serum & Pyridinoline (PYD) & Urine \\
Procollagen type 1 N-terminal propeptide (P1NP) & Serum/plasma & Deoxypyridinoline (DPD) & Urine \\
Osteocalcin (OC) & Serum/plasma & C-terminal crosslinked telopeptide (CTX) & Urine and serum/plasma \\
Bone Alkaline Phosphatase (BALP) & Serum & N-terminal crosslinked telopeptide (NTX) & Urine and serum/plasma \\
& & Tartrate resistant acid phosphatase -5b (TRACP-5b) & Serum \\
\hline
\end{tabular}


urine NTX or plasma/serum CTX, are seen after 1 month of treatment and reach a plateau from 3 months onwards. Relative plasma or serum CTX reductions of $50-70 \%$ after 3 months of treatment have been reported for several bisphosphonates. ${ }^{111}$ The decrease in bone formation markers is delayed compared to bone resorption markers, reaching a plateau after 6-12 months of treatment. Anabolic agents such as Teriparatide (PTH analogue) stimulates bone turnover. Procollagen type 1 $\mathrm{N}$-terminal propeptide starts to increase as early as 1 month with a peak at 6 months. Increases of $>200 \%$ have been documented. ${ }^{114-116}$

The LSC for each marker should be derived in order to determine whether changes in BTMs are of clinical significance in an individual patient. ${ }^{117}$ Changes of $>30 \%$ for plasma or serum-based markers should be of clinical significance as these changes exceeds the LSC which is between $25 \%$ and $30 \%$ for these markers. On average, BTMs change by $50 \%$ following antifracture treatment, making it easier to use in monitoring treatment efficacy at earlier time points than BMD changes. Insufficient BTMs reduction after a period of antiresorptive therapy with oral bisphosphonates may indicate lack of drug absorption or issues surrounding patient adherence. In the individual patient, where the change in BTMs is equivocal, another measurement is recommended 3 months later as caution should be exercised before changing treatment on the basis of an insufficient BTM response. Sequential BTMs measurements should be obtained on more than two separate occasions before such a clinical decision is made. The variability of the BTMs has improved significantly in recent years, and we propose that these markers, particularly plasma CTX and Procollagen type $1 \mathrm{~N}$-terminal propeptide measured on automated platforms, can be used in routine clinical practice to assess treatment response.

\section{Take-home messages}

- Osteoporosis and osteoporosis-related fractures is a common disorder which affects 1 in 2 women and 1 in 5 men. It is a systemic skeletal disorder which occurs as a result of an imbalance between bone formation and bone resorption.

- The WHO- designed fracture risk assessment tool (FRAX) calculates the 10-year probability of an osteoporotic fracture. This is computed from multiple extra-skeletal clinical risk factors with and without BMD at the femoral neck. It provides an aid in the assessment of an individual's fracture risk and in determining whether therapeutic intervention is required.

- Osteoporosis is preventable and treatable. Treatment includes non-pharmacological as well as pharmacological intervention.

- Non-pharmacological intervention includes modification of general lifestyle factors such as smoking cessation, avoidance of heavy alcohol use, ensuring regular weight-bearing exercise and optimum calcium/vitamin D intake.

- Bisphosphonates and Denosumab are anti-resorptive agents. They decrease vertebral fractures as well as non-vertebral fractures. PTH analogues are anabolic and used in patients at very high risk of fracture who have failed therapy with anti-resorptives.

- Monitoring response to therapy is important. Bone turnover markers, particularly serum-based markers such as serum CTX and P1NP have a role to play in assessing treatment efficacy and compliance.

\section{SUMMARY}

In summary, osteoporosis is a multifactorial disease that is an important public health and financial problem, associated with increased mortality and morbidity. Recently released fracture risk prognostic tools incorporate $\mathrm{BMD}$ and other independent clinical risk factors to estimate an individual's absolute fracture risk, thus aiding treatment decisions. Management should address investigation for secondary causes of osteoporosis and correction of this where possible. Treatment should focus on both non-pharmacological and pharmacological measures. Bisphosphonates are currently recommended as first choice treatment to postmenopausal women and men aged more than 50 years, in accordance with the NICE guidelines, due to its cost effectiveness and broad spectrum fracture efficacy data. To date, there is no consensus on best practice when it comes to treatment monitoring. Both BMD and BTMs, when used appropriately, are clinically useful surrogates of antifracture effectiveness; however, limitations and benefits of both should be taken into account.

\section{Competing interests None.}

Provenance and peer review Commissioned; externally peer reviewed.

\section{REFERENCES}

1. Seeman E, Delmas PD. Bone quality - the material and structural basis of bone strength and fragility. N Engl J Med 2006;354:2250-61.

2. Raisz LG. Pathogenesis of osteoporosis: concepts, conflicts, and prospects. J Clin Invest 2005;115:3318-25.

3. Licata A. Bone density vs bone quality: What's a clinician to do? Cleve Clin J Med 2009:76:331-6.

4. http://www.iofbonehealthorg/facts-and-statistics.htm

5. Van Staa TP, Dennison EM, Leufkens $\mathrm{H}$, et al. Epidemiology of fractures in England and Wales. Bone 2001;29:517-22.

6. Dennison $\mathbf{E}$, Cole Z, Cooper $\mathrm{C}$, et al. Diagnosis and epidemiology of osteoporosis. Curr Opin Rheumatol 2005;17:456-61.

7. Burge RT. The cost of osteoporotic fractures in the UK: Projections for 2000-2020. J of Med Economic 2001:4:51.

8. Cooper C, Atkinson EJ, Jacobsen SJ, et al. Population-based study of survival afte osteoporotic fractures. Am J Epidemiol 1993;137:1001-5.

9. Center JR, Nguyen TV, Schneider D, et al. Mortality after all major types of osteoporotic fracture in men and women: an observational study. Lancet 1999;353:878-82

10. Tosteson AN, Gottlieb DJ, Radley DC, et al. Excess mortality following hip fracture: the role of underlying health status. Osteoporos Int 2007;18:1463-72.

11. Randell AG, Nguyen TV, Bhalerao N, et al. Deterioration in quality of life following hip fracture: a prospective study. Osteoporos Int 2000;11:460-6.

12. Haentjens $\mathbf{P}$, Magaziner J, Colón-Emeric CS, et al. Meta-analysis: Excess mortality after hip fracture among older women and men. Annals of Int Med 2010;152:380-90.

13. Bliuc D, Nguyen ND, Milch VE, et al. Mortality risk associated with low- trauma osteoporotic fracture and subsequent fracture in men and women. JAMA 2009;301:513-21.

14. Styrkarsdottir U, Halldorsson BV, Gretarsdottir S, et al. New sequence variants associated with bone mineral density. Nat Genet 2009:41:15-17.

15. Ralston SH. Uitterlinden AG. Genetics of osteoporosis. Endocr Rev 2010;31:629-62.

16. Richards JB, Kavvoura FK, Rivadeneira F, et al. Collaborative meta-analysis: associations of 150 candidate Genes with osteoporosis and osteoporotic fracture. Ann Intern Med 2009;151:528-37.

17. Bonjour JP, Theintz G, Law F, et al. Peak bone mass. Osteoporosis Int 1994:4:7-13

18. Bonjour JP, Chevalley T, Ferrari $\mathrm{S}$, et al. The importance and relevance of peak bone mass in the prevalence of osteoporosis. Salud Publica Mex 2009;51:S5-13.

19. Winsloe C, Earl S, Dennison EM, et al. Early life factors in pathogenesis of osteoporosis. Curr Osteoporos Rep 2009:7:140-4.

20. Hofbauer LC, Khosla S, Dunstan CR, et al. The roles of osteoprotegerin and osteoprotegerin ligand in the paracrine regulation of bone resorption. J Bone Miner Res 2000;15:2-12.

21. Glass DA, Bialek P, Ahn JD, et al. Canonical Wnt signaling in differentiated osteoblasts controls osteoclast differentiation. Dev Cell 2005;8:751-64.

22. Robling AG, Niziolek PJ, Baldridge LA, et al. Mechanical stimulation of bone in vivo reduces osteocyte expression of SOST/sclerostin. J Biol Chem 2008;283:5866-75.

23. Kearns AE, Khosla S, Kosteniuk PJ. Receptor activator of nuclear factor- $\kappa B$ ligand and osteoprotegerin regulation of bone remodelling in health and disease. Endocr Rev 2008;29:155-92. 
24. Pacifici RJ. Estrogen, cytokines, and pathogenesis of postmenopausal osteoporosis. J Bone Miner Res 1996;11:1043-51.

25. Khosla S, Peterson JM, Egan K, et al. Circulating cytokine levels in osteoporotic and normal women. J Clin Endocrinol Metab 1994;79:707-11.

26. Rosen CJ, Donahue LR, Hunter SJ. Insulin-like growth factors and bone: the osteoporosis connection. Proc Soc Exp Biol Med 1994;206:83-102.

27. Cummings SR, Browner WS, Bauer D, et al. Endogenous hormones and the risk of hip and vertebral fractures among older women. Study of Osteoporotic Fractures Research Group. N Engl J Med 1998;339:733-8.

28. Greendale GA, Edelstein S, Barrett-Connor E. Endogenous sex steroids and bone mineral density in older women and men: The Rancho Bernardo Study. J Bone Miner Res 1997;12:1833-43.

29. Melton LJ III, Khosla S, Atkinson EJ, et al. Relationship of bone turnover to bone density and fractures. J Bone Miner Res 1997;12:1083-91.

30. Garnero P, Sornay-Rendu E, Chapuy MC, et al. Increased bone turnover in late postmenopausal women is a major determinant of osteoporosis. $J$ Bone Miner Res 1996:11:337-49.

31. Feik SA, Thomas CDL, Clement JG. Age-related changes in cortical porosity of the midshaft of the human femur. J Anat 1997;191:407-16.

32. Eisman J, Clapham S, Kehoe L. Osteoporosis prevalence and levels of treatment in primary care: the Australian BoneCare Study. J Bone Miner Res 2004;19:1969-75.

33. Siris ES, Chen YT, Abbott TA, et al. Bone mineral density thresholds for pharmacological intervention to prevent fractures. Arch Intern Med 2004;164:1108-12.

34. Kanis JA, Melton LJ III, Christiansen C, et al. The diagnosis of osteoporosis. J Bone Miner Res 1994:9:1137-41.

35. http://www.iscd.org/visitors/positions/officialpositionstext.cfm

36. Nguyen ND, Eisman JA, Center JR, et al. Risk factors for fracture in nonosteoporotic men and women. J Clin Endocrinol Metab 2007;92:955-62.

37. Sanders KM, Nicholson GC, Watts JJ, et al. Half the burden of fragility fractures in the community occur in women without osteoporosis. When is fracture prevention cost-effective? Bone 2006;38:694-700.

38. Cummings SR, Nevitt MC, Browner WS, et al. Risk factors for hip fracture in white women. Study of osteoporotic fractures research group. N Engl J Med 1995; 332:767-73.

39. Nguyen ND, Pongchaiyakul C, Center JR, et al. Identification of high-risk individuals for hip fracture: a 14-year prospective study. J Bone Miner Res 2005:20:1921-8.

40. Kanis JA, Borgstrom F, De Laet C, et al. Assessment of fracture risk. Osteoporos Int 2005:16:581-9.

41. Nguyen ND, Frost SA, Center JR, et al. Development of a nomogram for individualizing hip fracture risk in men and women. Osteoporos Int 2007:18:1109-17.

42. Kanis JA, Johnell 0 , Oden A, et al. FRAX and the assessment of fracture probability in men and women from the UK. Osteoporos Int 2008;19: 385-97.

43. Feskanich D, Willett W, Colditz G. Walking and leisure-time activity and risk of hip fracture in postmenopausal women. JAMA 2002;288:2300-6.

44. Gregg EW, Cauley JA, Seeley DG, et al. Physical activity and osteoporotic fracture risk in older women. Study of Osteoporotic Fractures Research Group. Ann Intern Med 1998:129:81-8.

45. Bonaiuti D, Shea B, lovine $\mathrm{R}$, et al. Exercise for preventing and treating osteoporosis in postmenopausal women. Cochrane Database Syst Rev 2009; (1):1-37.

46. Gillespie LD, Gillespie WJ, Robertson MC, et al. Interventions for preventing falls in elderly people. Cochrane Database Syst Rev 2003;(4):CD000340.

47. Day L, Fildes B, Gordon I, et al. Randomised factorial trial of falls prevention among older people living in their own homes. BMJ 2002;325:128-34.

48. Lips $\mathbf{P}$. Vitamin D deficiency and secondary hyperparathyroidism in the elderly: consequences for bone loss and fractures and therapeutic implications. Endocr Rev 2001;22:477-501.

49. Tang BM, Eslick GD, Nowson C, et al. Use of calcium or calcium in combination with vitamin $D$ supplementation to prevent fractures and bone loss in people aged 50 years and older: a meta-analysis. Lancet 2007:370:657-66.

50. Inderjeeth CA, Glennon D, Petta $A$, et al. Vitamin $D$ and muscle strength in patients with previous fractures. N Z Med J 2007;120:U2730.

51. Flicker L, Mead K, Maclnnis RJ, et al. Serum Vitamin D and falls in older women in residential care in Australia. J Am Geriatr Soc 2003;51:1533-8.

52. Boonen S, Lips P, Bouillon R, et al. Need for additional calcium to reduce the risk of hip fracture with vitamin D supplementation: evidence from a comparative metaanalysis of randomized controlled trials. J Clin Endocrinol Metab 2007; 92:1415-23.

53. Bischoff-Ferrari HA, Giovannucci E, Willett WC, et al. Estimation of optimal serum concentrations of 25-hydroxyvitamin d for multiple health outcomes. Am J Clin Nutr 2006;84:18-28.

54. Dawson-Hughes B, Heaney RP, Holick MF, et al. Estimates of optimal vitamin D status. Osteoporos Int 2005;16:713-16.

55. Bischoff-Ferrari HA, Dawson Hughes B, Baron JA, et al. Positive association between 25-hydroxy vitamin $D$ levels and bone mineral density: a population-based study of younger and older adults. Am J Med 2004;116:634-9.

56. Bischoff-Ferrari HA, Willett WC, Wong JB, et al. Fracture prevention with vitamin D supplementation: a meta-analysis of randomized controlled trials. JAMA 2005;293:2257-64
57. Heaney RP, Dowell MS, Hale CA, et al. Calcium absorption varies within the reference range for serum 25-hydroxyvitamin D. J Am Coll Nutr 2003;22 142-6.

58. Priemel M, von Domarus C, Klatte T0, et al. Bone mineralization defects and vitamin $D$ deficiency: histomorphometric analysis of iliac crest bone biopsies and circulating 25-hydroxyvitamin D in 675 patients. J Bone Miner Res 2010:25:305-12.

59. Lanham-New SA, Buttriss JL, Miles LM, et al. Proceedings of the rank forum on Vitamin D. Br J Nutr 2011:105:144-56.

60. Bolland MJ, Avenell A, Baron JA, et al. Effect of calcium supplements on risk of myocardial infarction and cardiovascular events: meta-analysis. BMJ 2010;341 c3691.

61. Giovannucci E, Liu Y, Hollis BW, et al. 25-Hydroxyvitamin D and risk of myocardial infarction in men: a prospective study. Arch Intern Med 2008:168:1174-80.

62. Autier $\mathbf{P}$, Gandini S. Vitamin D supplementation and total mortality. Arch Intern Med 2007;167:1730-7.

63. Vieth R. Vitamin D supplementation, 25-hydroxyvitamin D concentrations, and safety. Am J Clin Nutr 1999;69:842-56.

64. Heaney RP, Davies KM, Chen TC, et al. Human serum 25-hydroxycholecalciferol response to extended oral dosing with cholecalciferol. Am J Clin Nutr 2003; 77:204-10.

65. MacLean C, Newberry S, Maglione M, et al. Systematic review: comparative effectiveness of treatments to prevent fractures in men and women with low bone density or osteoporosis. Ann Intern Med 2008;148:197-213.

66. Rossouw JE, Anderson GL, Prentice RL, et al. Risks and benefits of estrogen plus progestin in healthy postmenopausal women: principal results From the Women's Health Initiative randomized controlled trial. JAMA 2002;288:321-33.

67. Bone HG, Hosking D, Devogelaer JP, et al. Ten Years' experience with alendronate for osteoporosis in postmenopausal women. $N$ Engl J Med 2004;350:1189-99.

68. Mellström DD, Sörensen $\mathrm{OH}$, Goemaere S, et al. Seven years of treatment with risedronate in women with postmenopausal osteoporosis. Calcf Tissue Int 2004; 75:462-8

69. Lyles KW, Colón-Emeric CS, Magaziner JS, et al. The effect of once-yearly Zoledronic acid $5 \mathrm{mg}$ on new fractures and mortality after hip fracture: The HORIZON Recurrent Fracture Trial. N Engl J Med 2007;357:266-81.

70. Black DM, Delmas PD, Eastell R, et al. Once-yearly zoledronic acid for treatment of postmenopausal osteoporosis. N Engl J Med 2007;356:1809-22.

71. Khosla S, Burr D, Cauley J, et al. Bisphosphonate-associated osteonecrosis of the jaw: report of a task force of the American Society for Bone and Mineral Research. J Bone Miner Res 2007;22:1479-91.

72. Pazianas $\mathbf{M}$, Miller $\mathbf{P}$, Blumentals WA et al. A review of the literature on osteonecrosis of the jaw in patients with osteoporosis treated with oral bisphosphonates: prevalence, risk factors, and clinical characteristics. Clin Ther 2007;29:1548-58.

73. Shane E. Evolving data about subtrochanteric fractures and bisphosphonates. N Engl J Med 2010;62:1825-7.

74. Odvina CV, Zerwekh JE, Rao DS, et al. Severely suppressed bone turnover: a potential complication of alendronate. J Clin Endocrinol Metab 2005:90:1294-301.

75. Abrahamsen B, Eiken P, Eastell R. Subtrochanteric and diaphyseal femur fractures in patients treated with alendronate: a register-based national cohort study. J Bone Miner Res 2009;24:1095-102.

76. Black DM, Kelly MP, Genant HK, et al. Bisphosphonates and fractures of the subtrochanteric or diaphyseal femur. N Engl J Med 2010;362:1761-71.

77. Green J, Czanner G, Reeves G, et al. Oral bisphosphonates and risk of cancer of oesophagus, stomach, and colorectum: case-control analysis within a UK primary care cohort. BMJ 2010;341

78. Cardwell CR, Abnet CC, Cantwell MM, et al. Exposure to oral bisphosphonates and risk of esophageal cancer. JAMA 2010;304:657-63.

79. Black DM, Schwartz AV, Ensrud KE, et al. Effects of continuing or stopping alendronate after 5 years of treatment: the Fracture Intervention Trial Long-term Extension (FLEX) :a randomized trial. JAMA 2006;296:2927-38.

80. Russell RG, Watts NB, Ebetino FH, et al. Mechanisms of action of bisphosphonates: similarities and differences and their potential influence on clinica efficacy. Osteoporos Int 2008;19:733-59.

81. Watts NB, Chines A, Olszynski $S$, et al. Fracture risk remains reduced one year after discontinuation of risedronate. Osteoporos Int 2008;19:365-72.

82. Watts NB, Diab DL. Long-term use of bisphosphonates in osteoporosis. J Clin Endocrinol Metab 2010;95:1555-65.

83. Luckey M, Kagan R, Greenspan S, et al. Once-weekly alendronate $70 \mathrm{mg}$ and raloxifene $60 \mathrm{mg}$ daily in the treatment of postmenopausal osteoporosis. Menopause 2004;11:405-15.

84. Smith MR, Fallon MA, Lee $\mathrm{H}$, et al. Raloxifene to prevent gonadotrophin-releasing hormone agonist-induced bone loss in men with prostate cancer: a randomized controlled trial. J Clin Endocrinol Metab 2004;89:3841-6.

85. Blake GM, Fogelman I. Theoretical model for the interpretation of BMD scans in patients stopping strontium ranelate treatment. J Bone Miner Res 2006;21:1417-24.

86. O'Donnell S, Cranney A, Wells G, et al. Strontium ranelate for preventing and treating postmenopausal osteoporosis. Cochrane Database Syst Rev 2006;3: CD005326. 
87. Jerome CP, Burr DB, Van Bibber $T$, et al. Treatment with human parathyroid hormone (1-34) for 18 months increases cancellous bone volume and improves trabecular architecture in ovariectomized cynomolgus monkeys (Macaca fascicularis). Bone 2001:28:150-9.

88. Neer RM, Arnaud CD, Zanchetta JR, et al. Effect of parathyroid hormone (1-34) on fractures and bone mineral density in postmenopausal women with osteoporosis. N Engl J Med 2001;344:1434-41.

89. Greenspan SL, Bone HG, Ettinger MP, et al. Effect of recombinant human parathyroid hormone $(1-84)$ on vertebral fracture and bone mineral density in postmenopausal women with osteoporosis: a randomized trial. Ann Intern Med 2007; 146:326-39.

90. Modelska K, Cummings S. Tibolone for postmenopausal women: systematic review of randomized trials. J Clin Endocrinol Metab 2002;87:16-23.

91. Cummings SR, Ettinger B, Delmas PD, et al. The effects of tibolone in older postmenopausal women. N Engl J Med 2008;359:697-708.

92. Katznelson L, Finkelstein JS, Schoenfeld DA, et al. Increase in bone density and lean body mass during testosterone administration in men with acquired hypogonadism. J Clin Endocrinol Metab 1996;81:4358-65.

93. Behre HM, Kliesch S, Leifke $\mathrm{E}$, et al. Long-term effect of testosterone therapy on bone mineral density in hypogonadal men. J Clin Endocrinol Metab 1997:82:2386-90.

94. Amory JK, Watts NB, Easley KA, et al. Exogenous testosterone or testosterone with finasteride increases bone mineral density in older men with low serum testosterone. J Clin Endocrinol Metab 2004:89:503-10.

95. McClung MR, Lewiecki EM, Cohen SB, et al. Denosumab in postmenopausal women with low bone mineral density. N Engl J Med 2006;354:821-31.

96. Cummings SR, San Martin J, McClung MR, et al. Denosumab for prevention of fractures in postmenopausal women with osteoporosis. N Engl J Med 2009:361:756-65.

97. Li X, Ominsky MS, Warmington KS, et al. Sclerostin antibody treatment increases bone formation, bone mass, and bone strength in a rat model of postmenopausal osteoporosis. J Bone Miner Res 2009;24:578-88.

98. Murphy MG, Cerchio K, Stoch SA, et al. Effect of L-000845704, an alphaVbeta3 integrin antagonist, on markers of bone turnover and bone mineral density in postmenopausal osteoporotic women. J Clin Endocrinol Metab 2005:90:2022-8.

99. Vasiljeva 0, Reinheckel T, Peters C, et al. Emerging roles of cysteine cathepsins in disease and their potential as drug targets. Curr Pharm Des 2007:13:387-403.

100. Sarkar S, Mitlak BH, Wong M, et al. Relationships between bone mineral density and incident vertebral fracture risk with raloxifene therapy. J Bone Miner Res 2002:17:1-10.

101. Wasnich RD, Miller PD. Antifracture efficacy of antiresorptive agents are related to changes in bone density. J Clin Endocrinol Metab 2000;85:231-6.

102. Hochberg MC, Greenspan S, Wasnich RD, et al. Changes in bone density and turnover explain the reductions in incidence of non-vertebral fractures that occur during treatment with antiresorptive agents. J Clin Endocrinol Metab 2002;87:1586-92.
103. Cummings SR, Karpf DB, Harris F, et al. Improvement in spine bone density and reduction in risk of vertebral fractures during treatment with antiresorptive drugs. Am J Med 2002;112:281-9.

104. Binkley N, Bilezikian JP, Kendler DL, et al. Official positions of the international society for clinical densitometry and executive summary of the 2005 position development conference. J Clin Densitom 2006:9:4-14.

105. Seibel MJ, Lang M, Geilenkeuser WJ. Interlaboratory variation of biochemical markers of bone turnover. Clin Chem 2001;47:1443-50.

106. Garnero P, Borel 0, Delmas PD. Evaluation of a fully automated serum assay for C-terminal cross-linking telopeptide of type I collagen in osteoporosis. Clin Chem 2001;47:694-702

107. Claudon A, Vergnaud P, Valverde C, et al. New automated multiplex assay for bone turnover markers in osteoporosis. Clin Chem 2008;54:1554-63.

108. Glover SJ, Garnero P, Naylor K, et al. Establishing a reference range for bone turnover markers in young, healthy women. Bone 2008;42:623-30.

109. Bauer DC, Black DM, Garnero P, et al. Change in bone turnover and hip, non-spine and vertebral fracture in alendronate-treated women: the fracture intervention trial. J Bone Miner Res 2004;19:1250-8.

110. Eastell R, Christiansen C, Grauer A, et al. Effects of denosumab on bone turnover markers in postmenopausal osteoporosis. J Bone Miner Res. Published Online First: 13 September 2010

111. Rosen CJ, Hochberg MC, Bonnick SL, et al. Treatment with once-weekly alendronate $70 \mathrm{mg}$ compared with once-weekly risedronate $35 \mathrm{mg}$ in women with postmenopausal osteoporosis: a randomised double-blind study. J Bone Miner Res 2005;20:141-51.

112. Delmas PD. Markers of bone turnover for monitoring treatment of osteoporosis with anti-resorptive drugs. Osteoporos Int 2000;11(Suppl 6): S66-76.

113. Meunier PJ, Roux C, Seeman E, et al. The effects of strontium ranelate on the risk of vertebral fractures in women with postmenopausal osteoporosis. N Engl J Med 2004:350:459-68.

114. Bauer DC, Garnero P, Bilezikian JP, et al. Short-term changes in bone turnover markers and bone mineral density response to parathyroid hormone in postmenopausal women with osteoporosis. J Clin Endocrinol Metab 2006;91:1370-5.

115. Dobnig H, Sipos A, Jiang Y, et al. Early changes in biochemical markers of bone formation correlate with improvements in bone structure during teriparatide therapy. J Clin Endocrinol Metab 2005;90:3970-7.

116. Black DM, Greenspan SL, Ensrud KE, et al. The effects of parathyroid hormone and alendronate alone or in combination in postmenopausal osteoporosis. N Engl J Med 2003;349:1207-15

117. Lewiecki EM. Benefits and limitations of bone mineral density and bone turnover markers to monitor patients treated for osteoporosis. Curr Osteoporos Rep 2010;8:15-22. 